\title{
Undifferentiated Carcinoma of Pancreas Presenting as Obstructive Jaundice
}

\author{
A Sekaran ${ }^{1}$, S Koppula ${ }^{1}$, DN Reddy ${ }^{2}$, S Lakhtakia ${ }^{2}$, B Patodiya $^{3}$ \\ Departments of ${ }^{1}$ Pathology and ${ }^{2}$ Gastroenterology and ${ }^{3}$ Oncology, AIG Hospitals, Hyderabad, Telangana, India.
}

\section{Corresponding Author: \\ Dr Anuradha Sekaran \\ Email: dr.sanuradha@aighospitals.com \\ This is an Open Access article distributed under the terms of the Creative Commons Attribution License (creativecommons.org licenses/by/3.0).}

Received

Accepted

November 27, 2020

Published

July 20, 2021

\begin{abstract}
Background: Undifferentiated carcinoma of pancreas (UCP) is a rare neoplasm that usually occurs in elderly patient. Case Report: We report a case of an undifferentiated carcinoma of pancreas (UCP) in a young male who presented with short history of painless progressive jaundice, dyspnea, dry cough and recent weight loss. Imaging studies (CECT) revealed an ill-defined hypo-enhancing mass noted in the pancreatic head region encasing vessels. Histology obtained by EUS guided fine needle biopsy was consistent with undifferentiated carcinoma of pancreas. Conclusion: Undifferentiated carcinoma of pancreas is a rare pancreatic neoplasm carrying poor prognosis.
\end{abstract}

Keywords: Carcinoma, Jaundice, Male, Pancreas, Pancreatic Neoplasms.

\section{Introduction}

Pancreatic carcinoma is the second most common gastrointestinal malignancies after colorectal carcinomas [1]. Undifferentiated carcinoma of the pancreas (UCP) is rare group of aggressive malignant epithelial neoplasm accounting for $2-7 \%$ of all pancreatic tumors [2]. Historically it has four variants: anaplastic, sarcomatoid, rhabdoid and carcinosarcoma [3]. A separate category, undifferentiated pancreatic carcinoma with osteoclast-like giant cells (UC-OGC) - was first reported by Rosai in 1968 [4]. UCP carry poorer prognosis compared to UC-OGC and typical ductal adenocarcinoma of the pancreas [5].

\section{Case Report}

A 21-year-young man presented with painless jaundice, dyspnea and dry cough for 10 days. He reported significant weight loss over last one month. Laboratory tests showed mild elevation of total bilirubin and slight increase in transaminase and alkaline phosphatase, $135 \mathrm{U} / \mathrm{L}$ (normal 40120 U/L). Serum CA-19.9 and CEA were normal. Contrast enhanced $\mathrm{CT}$ scan of abdomen revealed a large $6.6 \times 4.3 \times 3.5 \mathrm{~cm}$ ill-defined solid hypoenhancing mass in the pancreatic head encasing the common hepatic artery, gastroduodenal artery and partly encasing main portal vein [Fig.1]. There was loss of fat planes between the mass and periportal soft tissue mass. Additional findings were hepatomegaly and mild bilateral pleural effusion. An endoscopic ultrasound (EUS) guided biopsy was performed using $22 \mathrm{G}$ core needle (Acquire, Boston Scientific) via duodenum from

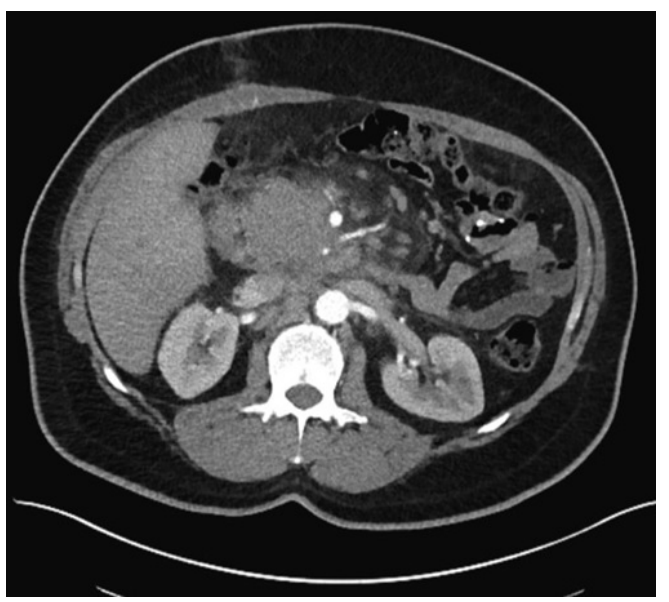

Fig.1: CT scan of abdomen: Large mass in head of the pancreas with vascular encasement. 
the pancreatic mass. Microscopy showed a cellular neoplasm composed of non-cohesive, pleomorphic, and bizarre cells without any glandular formation [Fig.2a]. Cells were round-to-oval with vesicular to hyperchromatic nuclei, inconspicuous nucleolus and scant to moderate cytoplasm. The cells show marked atypia. There were occasional multinucleated giant cells. There were no lymphovascular emboli, or perineural invasion spindle cells, sarcomatoid or osteoid changes [Fig.2b-d]. The surrounding fibrous stroma was scant (in contrast to conventional invasive pancreatic ductal adenocarcinoma). No transition between adenocarcinoma and undifferentiated components was observed. Immunohistochemically, most neoplastic cells were positive for IMP3 [Fig.3a]. IHC for mCEA, EMA and pancytokeratin were negative. Other markers like synaptophysin, chromogranin, CD 56, CD20, LCA, CD30, SALL4, s-100, HMB-45 and CA 19-9 were also negative in the tumor cells ruling out several differential diagnoses like neuroendocrine tumor, lymphoma, germ cell tumor and melanoma. Focal macrophages showed immuno-reactive for LCA and CD68 [Fig.3b,c]. The final diagnosis was an undifferentiated carcinoma of the pancreas. Due to logistic limitations ancillary techniques like ex vivo chemo sensitivity or next generation-based mutation analysis was not done in this case

He is presently initiated on palliative intent chemotherapy with paclitaxel (175 mg per meter square) and carboplatin (AUC5) on three weekly cycle. He will be evaluated after three months of treatment by an interim CT scan.

\section{Discussion}

Undifferentiated carcinoma of pancreas with/ without osteoclast-like giant cells is a rare epithelial neoplasm composed of pleomorphic mononuclear cells without any glandular structure or other features to indicate a definite differentiation. The histogenesis of these cellular populations remains unclear. Authors have favored either
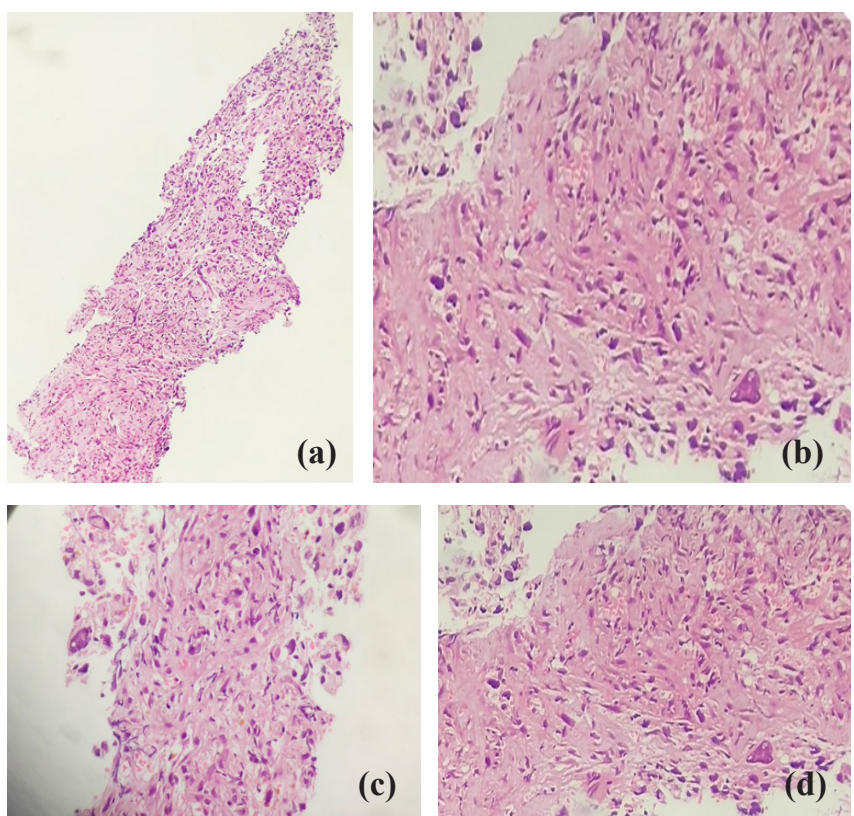

Fig.2 (a): FNB core shows atypical cells $(H \& E \times 100)$; (b): Pleomorphic cells arranged in non-cohesive pattern (H\&E $\times 200)$; (c): Pleomorphic and bizzare cells scattered in noncohesive pattern $(H \& E \times 200)$; (d): Pleomorphic cells with round to oval cells, hyperchromatic nuclei. Marked atypia noted $(H \& E \times 400)$.
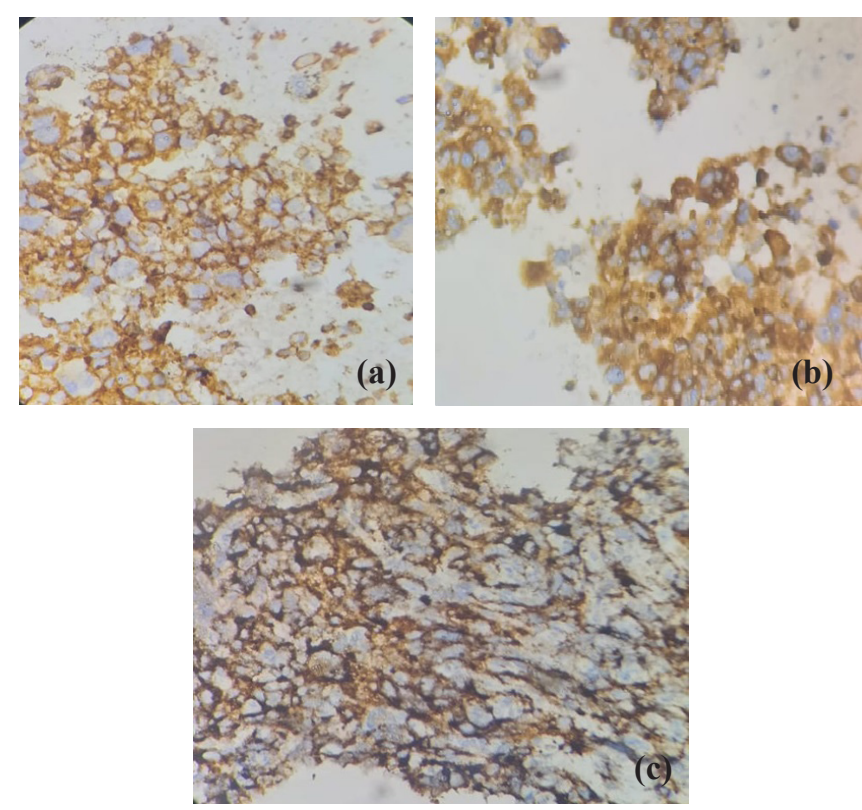

Fig.3 (a): IMP3 positive atypical cells $(I H C \times 200)$; (b): CD45 highlights few lymphocytes (IHC $\times 400)$; (c): CD68 positive macrophages and few multinucleated giant cells. $(I H C \times 200)$. 
mesenchymal or epithelial origin [6,7]. Luchini et al. [8], suggested that UC-OGC's are variants of pancreatic ductal adeno-carcinoma (PDAC) due to the presence of shared mutations in KRAS and other critical tumor suppressor genes commonly related to PDAC (TP53, CDKN2A and SMAD4).

UCP is more common in elderly age patients. The average age is 63-70 years with both genders being affected equally [6]. The common symptoms reported are abdominal or back pain (in two-thirds), painless jaundice (in one-third) and half the cases show weight loss [6,9]. Elevated serum CA19-9 levels are seen in two-third cases [6]. UCP is invasive cancer and carries a poor prognosis. In our case, patient was a $21 \mathrm{yr}$ old male and presented with painless jaundice, dyspnea and weight loss with normal CA19-9 levels. Commonly UCP occurs in the body and tail of the pancreas $(50-70 \%)[6,9]$. The index case presented as a large ill-defined mass in the head of pancreas. The mass lesion was solid, hypo-enhancing and had loss of fat planes while others observed heterogeneous mass with hyper and hypoechoic regions [1].

Undifferentiated carcinoma pancreas with osteoclast-like giant cells (UC-OGC) comprise of benign giant cells in a background of atypical pleomorphic mononuclear malignant cells [10]. It is hypothesized that OGC recruitment is a result of chemotactic factors produced by neoplastic cells [9]. These giant cells are typically arranged in nodules, sheets or are singly scattered and associated with areas of hemorrhage. Some cases show presence of metaplastic and mature bone tissue. Few show associated pancreatitis and pancreatic intra-epithelial lesions [6]. In our case, the above observations were absent. UCP usually shows lymph-vascular and perineural invasion. Muraki et al. [6] series had lymph-vascular in 63\% and perineural invasion in $32 \%$ (compared to $86 \%$ in PDAC [6]. The given core biopsy had not showed lymph-vascular or perineural invasion. Muraki et al. [6] observed lymph node metastasis in UCOGC was significantly less compared to PDAC
(23\% vs 64\%) [6]. In our case, atypical pleomorphic cells were immunoreactive for IMP3 and negative for PANCK, EMA, mCEA, CA19.9. Negative LCA, CD20, CD30 and SALL4 ruled out lymphoma and germ cell tumor. Other differential diagnosis like neuroendocrine tumor and melanoma were ruled out with negative synaptophysin, chromogranin, CD56, S-100 and HMB-45 respectively. Occasional giant cells were immunoreactive for CD68 and LCA. The differential diagnoses also included cystadenocarcinomas [6].

Surgical resection is preferred treatment [11]. UCP has poor prognosis. Median survival reported is 5-7 months. For adenocarcinoma of pancreas FOLFIRINOX or Gemcitabine with nab paclitaxel is considered as category I chemotherapy. However due to rarity of undifferentiated carcinoma of pancreas there is no category I regimen, however often the choice of regimen is extrapolated from adenocarcinoma trials. Few centres also choose chemotherapy based on ex vivo chemo-sensitivity with excellent response [12]. In the present case initial chemotherapy choice is made based on stage, performance status and age and travelling logistics. Paclitaxel and carboplatin are chosen by extrapolating response from other undifferentiated malignancies and while minimising hospital visit (patient being from outstation and due to covid-19 pandemic). In case of response, 6 cycles of same chemotherapy are planned. In case of nonresponse or progression of disease, $2^{\text {nd }}$ choice of chemotherapy is planned to be FOLFIRINOX. Being an aggressive regimen, patient need to be in good performance status at time of progression. In UCP most common clinical response is partial and median overall survival is around 6 months [13].

\section{Conclusion}

Undifferentiated carcinoma of pancreas is a rare pancreatic neoplasm carrying poor prognosis. This tumor can display various clinical characteristics. Its histogenesis is controversial. UCP with osteoclast-like giant cell carry better prognosis 
when compared to carcinoma without osteoclastlike giant cells. Due to its rarity, and poor prognosis, therapeutic guidelines are limited. Further studies may help establish treatment modalities and possible molecular biomarkers.

Contributors: AS: manuscript writing, histopathology; SK, DNR, SL: manuscript editing, patient management; SK: critical inputs into the manuscript and histopathology. AS will act as a study guarantor. All authors approved the final version of this manuscript and are responsible for all aspects of this study.

Funding: None; Competing interests: None stated.

\section{References}

1. Moore JC, Bentz JS, Hilden K, Adler DG. Osteoclastic and pleomorphic giant cell tumors of the pancreas: A review of clinical, endoscopic, and pathologic features. World J Gastrointest Endosc. 2010;2:15-19.

2. Paal E, Thompson LD, Frommelt RA, Przygodzki RM, Heffess CS. A clinicopathologic and immunohistochemical study of 35 anaplastic carcinomas of the pancreas with a review of the literature. Ann Diagn Pathol. 2001;5:129-140.

3. Agaimy A, Haller F, Frohnauer J, Schaefer IM, Ströbel P, Hartmann A, Pancreatic undifferentiated rhabdoid carcinoma: KRAS alterations and SMARCB1 expression status define two subtypes Mod Pathol. 2015;28(2):248260.

4. Rosai J. Carcinoma of pancreas simulating giant cell tumor of bone: electron-microscopic evidence of its acinar cell origin. Cancer. 1968;22:333-344.

5. Pan ZG, Wang B. Anaplastic carcinoma of the pancreas associated with a mucinous cystic adenocarcinoma.
A case report and review of the literature. J Pancreas. 2007;8:775-782.

6. Muraki T1, Reid MD, Basturk O, Jang KT, Bedolla $\mathrm{G}$, Bagci $\mathrm{P}$, et al. Undifferentiated carcinoma with osteoclastic giant cells of the pancreas. Am J Surg Pathol. 2016;40(9):1203-1216.

7. Maksymov V, Khalifa MA, Bussey A, Carter B, Hogan M. Undifferentiated (anaplastic) carcinoma of the pancreas with osteoclast-like giant cells showing various degree of pancreas duct involvement: a case report and literature review. J Pancreas. 2011;12(2):170-176.

8. Luchini C, Pea A, Lionheart G, Mafficini A, Nottegar A, Veronese N, et al. Pancreatic undifferentiated carcinoma with osteoclast-like giant cells is genetically similar to, but clinically distinct from, conventional ductal adenocarcinoma. J Pathol. 2017;243(2):148-154.

9. Sah SK, Li Y, Li Y. Undifferentiated carcinoma of the pancreas with osteoclast-like giant cells: a rare case report and review of the literature. Int J Clin Exp Pathol. 2015;8(9):11785-11791.

10. Jo S. Huge undifferentiated carcinoma of the pancreas with osteoclast-like giant cells. World J Gastroenterol. 2014;20(10):2725-2730.

11. Hur YH, Kim HH, Seoung JS, Seo KW, Kim JW, Jeong YY, et al. Undifferentiated carcinoma of the pancreas with osteoclast-like giant cells. J Korean Surg Soc. 2011;81(2):146-150.

12. Wakatsuki T1, Irisawa A, Imamura H, Terashima M, Shibukawa G, Takagi T. Complete response of anaplastic pancreatic carcinoma to paclitaxel treatment selected by chemosensitivity testing. Int J Clin Oncol. 2010;15:310313.

13. Clark CJ, Graham RP, Arun JS, Harmsen WS, Reid-Lambardo KM. Clinical outcomes for anapalstic pancreatic cancer: A population based study. J Am Coll Surg. 2012;215:627-634. 